\title{
Buenas Prácticas (Códigos de) Best Practices (Codes of)
}

\author{
Txetxu Ausín* \\ Instituto de Filosofía, CSIC \\ txetxu.ausin@,cchs.csic.es
}

Recibido / received: 17/04/2018

Aceptado / accepted: 30/07/2018

DOI: https://doi.org/10.20318/eunomia.2018.4354

\section{Resumen}

Los códigos de buenas prácticas recogen de manera ordenada y sistemática aquellas experiencias que resultan aconsejables en un determinado ámbito porque han arrojado resultados positivos y han demostrado su éxito y utilidad, de modo que merece la pena que se repitan y sean compartidas. Especialmente relevantes en el ámbito de las organizaciones del sector público, se mueven entre la tradición y la innovación y entre sus funciones están el reconocimiento de la misión de la actividad, la generación de conocimiento, el aprendizaje e innovación sobre la misma, la protección frente a presiones externas, la prevención de malas prácticas, la confianza y la ejemplaridad. Hay discusión sobre su valoración pero, como mínimo, deberían responder a un serie de requisitos: participación, flexibilidad, adhesión, accesibilidad, monitorización y difusión.

\section{Palabras clave}

Buena práctica/mala práctica, modelo, ética, innovación, cultura organizacional.

\begin{abstract}
The codes of best practices collect in an orderly and systematic way those experiences that are advisable in a certain field because they have yielded positive results and have shown their success and usefulness so that they are worth repeating and sharing. Especially relevant in the realm of public sector organizations, they move between tradition and innovation and among their functions are the recognition of the mission of the activity, the generation of knowledge, learning and innovation about it, protection against external pressures, the prevention of bad practices, trust, and exemplariness. There is discussion about their assessment but, at a minimum, they should respond to a series of requirements: participation, flexibility, adhesion, accessibility, monitoring and dissemination.
\end{abstract}

\section{Keywords}

Best practice/bad practice, model, ethic, innovation, organizational culture.

SUMARIO. 1. Definición. 2. Tradición e innovación. 3. Funciones. 4. Valoración. 5. Requisitos. 6. Conclusiones.

\footnotetext{
* Este trabajo se realiza en el marcos de los proyectos de investigación KONTUZ! "Responsabilidad causal de la comisión por omisión" (MINECO FFI2014-53926-R) y NEW TRUST-CM "Programa interuniversitario en cultura de la legalidad" (CONSEJERÍA DE EDUCACIÓN, COMUNIDAD DE MADRID S2015/HUM-3466).
} 


\section{Definición}

Las "buenas prácticas" se refieren a aquellas experiencias, procesos y actividades que resultan aconsejables en un determinado ámbito porque han arrojado resultados positivos y han demostrado su éxito y utilidad en un contexto concreto, de modo que merece la pena que se repitan y sean compartidas por el mayor número de implicados que puedan adoptarlas. Una definición estándar utilizada en el ámbito de la Unión Europea es la siguiente:

"[Buenas prácticas son] Fórmulas que han demostrado, por medio de la investigación y la evaluación, su eficacia y sostenibilidad, que producen resultados sobresalientes y que pueden ser aplicables y adaptables a otras situaciones" (Comisión Europea, 2004: 13).

El concepto de "buenas prácticas" se utiliza en una amplísima variedad de ámbitos y contextos para referirse a las formas óptimas de realizar un proceso o actividad y que, por ello, constituyen un modelo. En este sentido de "modelos", son prototipos ${ }^{1}$, que pueden ser sistematizados en códigos, a los que se les confiere un valor porque permiten aprender de las experiencias previas y aprendizajes en ese determinado ámbito.

La búsqueda de "buenas prácticas" se relaciona con el planteamiento de criterios de calidad y excelencia en las organizaciones y sus actividades, y remiten no solo a la gestión y los procedimientos, sino también a la satisfacción de los implicados y al más elevado cumplimiento de los bienes internos (principios) que caracterizarían dicha actividad.

En resumen, las "buenas prácticas" incluyen los siguientes atributos:

- Desarrollan soluciones positivas y de mejora de una actividad.

- Parten de una evaluación cuidadosa basada en evidencias y del análisis de los valores y principios de la actividad.

- Actúan como modelos y guías.

- Se sistematizan a través de su formulación en códigos u otros documentos, propiciando su institucionalización y sostenibilidad (manteniéndose en el tiempo y produciendo efectos duraderos).

- Generan un conocimiento compartido que implica su difusión y réplica.

En el contexto de la ética pública, de la ética de las organizaciones y actividades del sector público (Ausín, 2012; Villoria \& Izquierdo, 2015), los códigos de buenas prácticas son un instrumento fundamental de la infraestructura ética que persigue fomentar estándares de excelencia y calidad en dichas actividades. Los códigos de buenas prácticas en el sector público son declaraciones sobre valores, principios y conductas deseables y exigibles en las organizaciones del sector público, que presentan diferentes niveles de generalidad y de especificidad. Se proponen como prototipos de buena conducta y buenos procedimientos en dicho ámbito y promueven la excelencia profesional y los bienes internos (principios) de la actividad, como son la integridad, la honestidad, la imparcialidad y el respeto, entre otros. Los códigos de buenas prácticas en el ámbito del sector público recogen valores, 
responsabilidades, roles, obligaciones y restricciones aplicables en las organizaciones del sector público y lo hacen con un carácter eminentemente orientador (OCDE, 1997, 2000).

Los códigos se caracterizan porque no pretenden regular conductas legalmente estipuladas; va de suyo el cumplimiento de la ley y de los reglamentos aplicables a cada actividad del servicio público. Su pretensión es la de incidir en el ethos del servidor público, generando hábitos, convicciones y cambio de cultura institucional que, en última instancia, impregna a la misma sociedad, favoreciendo la confianza en las instituciones sociales.

\section{Tradición e innovación}

Aunque pueda parecer un contrasentido, los códigos de buenas prácticas se mueven entre la tradición y la innovación. Vamos a explicarnos.

Por un lado, existe una larguísima tradición histórica en el desarrollo y formulación de códigos éticos y de conducta en muchas actividades y ámbitos profesionales. Es bien conocido el caso del "Juramento Hipocrático", que orienta a los médicos en su actividad profesional y que se atribuye a Hipócrates o a un discípulo suyo en el siglo $\mathrm{V}$ a. C. Los así llamados "códigos deontológicos" definen los principios y conductas de actividades profesionales como la medicina, el periodismo, la ingeniería, la abogacía, etc. El término "deontología" remite a Jeremy Bentham y hace referencia al conjunto de principios y reglas de carácter no técnico que rigen el ejercicio de una profesión y que determinan, por tanto, la pertenencia a un grupo profesional; de ahí la estrecha vinculación de los códigos deontológicos con los colegios y agrupaciones profesionales. No deja de ser compleja la relación de los códigos deontológicos con la ética y, muy especialmente, con el derecho (Líbano, 2011). Sobre todo cuando van más allá de su carácter orientador y se convierten en obligaciones de necesario cumplimento para los profesionales sometidos a las mismas (sobre esto volveremos al final del artículo).

En el ámbito de la función pública podemos encontrar también un precedente de los códigos de buenas prácticas en los llamados "Tribunales de Honor"2. Los Tribunales de Honor son unas instituciones típicamente españolas, sin parangón en el Derecho extranjero, que nacen en el ámbito castrense para juzgar oficiales (Real Decreto de 3 de enero de 1867). Se extienden luego a la Administración pública (en la legislación de funcionarios civiles de 1918) y más tarde a la esfera privada, en especial a los colegios profesionales. Estaban formados por los pares del encausado y tenían por finalidad juzgar la dignidad de éste para pertenecer al cuerpo o profesión de la que era miembro. De resultar declarado indigno, el sujeto era expulsado del cuerpo, sin que pudiera interpone recurso alguno. Estos tribunales no jugaban actos aislados, sino conductas y estados de opinión acerca de la dignidad de un individuo para formar parte de un cuerpo. El bien jurídico protegido no era el honor del enjuiciado, sino el del cuerpo al que pertenecía. El procedimiento era sencillo, se daba audiencia al interesado y se mantenía casi clandestina la tramitación que concluía con la absolución o la separación del servicio y la consiguiente propuesta a la autoridad correspondiente. El artículo 95, párrafo último, de la Constitución española de 1931 abolió todos los tribunales de honor "tanto civiles como militares". Tras la Guerra Civil se repusieron con la Ley de Tribunales de Honor, de 17 de octubre de 1941. El sistema

2 Agradezco a Ángel Zurita, Letrado del Ayuntamiento de Bilbao y Vicepresidente de la Asociación de Letrados de Entidades Locales, su observaciones y comentarios sobre los Tribunales de Honor y la jugosa y larga charla mantenida acerca de la ética pública y los valores municipales, en un larguísimo y accidentado viaje que compartimos. 
se completó con la Ley de Funcionarios Civiles del Estado, de 7 de febrero de 1964. El artículo 26 de la Constitución española de 1978 prohibió los Tribunales de Honor en el ámbito de la Administración civil y de las organizaciones profesionales (y posteriormente se hizo lo mismo en el ámbito militar con la Ley Orgánica 5/2005, de 17 de noviembre, de la Defensa Nacional, que en su artículo 21.2 establece que quedan prohibidos los Tribunales de Honor en el ámbito militar).

Frente a este "peso" de la tradición con relación a los códigos de buenas prácticas, también hay que decir que, por otro lado, tienen un innegable carácter de innovación social en la medida en que desarrollan soluciones nuevas o creativas, aportan mejoras e incorporan capacidad de cuestionamiento, retroalimentación y reorientación de las actividades a las que se refieren. Recuérdese que las innovaciones se caracterizan por ser procesos interactivos que generan algo nuevo, transformador y valioso en sistemas y entornos determinados (Echeverría, 2017). Las innovaciones alteran los sistemas y entornos donde se producen. En este sentido, los códigos de buenas prácticas suscitan no pocas veces rechazo y resistencias (sin oposición, no hay innovación), precisamente porque conllevan cambios y a veces transformaciones profundas. Pensemos en el nuevo paradigma de gobierno abierto, de transparencia y de acceso a la información, considerado uno de los elementos fundamentales de las buenas prácticas de la administración pública contemporánea. O en el valor de la participación ciudadana desde una perspectiva de administración relacional (Alguacil, 2012). Y es que los códigos de buenas prácticas en el ámbito de las organizaciones del sector público adquieren el carácter de innovación social que surge de la experiencia, el uso y la interacción entre los servidores públicos y la ciudadanía, en el sentido de la "democratización de la innovaciones" Von Hippel (2005).

Podemos mencionar códigos de buenas prácticas en el ámbito de la función pública que van desde el Informe Northcote-Trevelyan de 1854 en Reino Unido al Civil Service Code (1996) del mismo país, pasando por la Ley de Ética en el Gobierno de USA (1978), la Public Service Act de Australia (1999) o el Código Ético y de Valores para el Sector Público de Canadá (2003), aparte de los códigos-tipo desarrollados por organizaciones internacionales como la ONU, la OCDE, el Consejo de Europa, el Centro Latinoamericano de Administración para el Desarrollo (CLAD) o la Comisión Europea, entre otros (Diego, 2013). En 2005, en España se aprobó el Código de Buen Gobierno de los miembros del Gobierno y de los altos cargos de la Administración General del Estado -derogado expresamente por la Ley 3/2015 de 30 de marzo, reguladora del ejercicio del alto cargo de la AGE, disposición derogatoria c)- y posteriormente el Estatuto Básico del Empleado Público (2007). También ha habido un importante desarrollo reciente de códigos de ética y buenas prácticas en el ámbito autonómico y municipal (Longo y Albareda, 2015; Jiménez, 2017b) -siendo el Sistema de Integridad Institucional de la Diputación Foral de Gipuzkoa el más avanzado hasta el momento, pues incluye no solo un código para altos cargos sino otros tres: uno para el empleo público foral, otro en materia de contratación pública y uno próximo para subvenciones: https://www.gipuzkoa.eus/es/diputacion/sistema-de-integridad ${ }^{3}$.

3 Además, otra de las singularidades de este Sistema de Integridad Institucional es que el órgano de garantía, la Comisión de Ética Institucional, está formada por tres miembros externos al gobierno foral, mientras que los internos con voz y voto se reducen a dos. 


\section{Funciones}

A la luz de todo lo anterior, cabe establecer algunas funcionalidades básicas de los códigos de buenas prácticas.

\subsection{Reconocimiento e identidad:}

Los códigos y documentos de buenas prácticas constituyen un reconocimiento público de la misión de una determinada actividad y, en el caso que nos ocupa, la caracterización de los valores, principios y prácticas que definen a las organizaciones del sector público. Remiten a una toma de conciencia de los compromisos no solo técnicos sino también morales en este ámbito, que no se reducen al combate contra la corrupción económica sino que también se refieren a otros valores y anti-valores implicados en el servicio público, como el abuso de autoridad, el acoso, la falsedad, el nepotismo, etc. Los códigos de buenas prácticas contemplan tanto valores morales indispensables, como el respeto, la honestidad o la integridad, como un conjunto de exigencias y competencias técnicas y organizativas. La "bondad" de las prácticas recogidas remiten a un principio ético básico de beneficencia, que se interpreta como hacer bien las cosas (eficiencia) para hacer el bien a los demás (ética).

Como poco, los códigos de buenas prácticas introducen reflexividad en la actividad a la que se refieren, constituyendo una meditación en voz alta, pública, sobre las dimensiones morales de esta actividad, sobre qué prácticas ayudan a alcanzar las metas de la misma y sobre qué valores y principios se consideran necesarios para proporcionar a la sociedad el bien interno que le incumbe, lo que le es intrínsecamente propio, su misión. De este modo, favorecen asimismo el sentimiento de pertenencia a una organización o institución, creando "identidad".

\subsection{Pedagogía}

Al establecer normas, obligaciones y buenas prácticas, los códigos funcionan como guías de conducta de la actividad. En este sentido, tienen una triple función: informativa, argumentativa y de aprendizaje. Generan un conocimiento compartido sobre la actividad, sirven como argumento en la discusión y reflexión sobre la misma y permiten el intercambio y la difusión de experiencias exitosas para su réplica. El objetivo último de los códigos de buenas prácticas es impulsar el cambio en la cultura de una determinada organización o servicio, de ahí su carácter innovador, como hemos dicho, forjando hábitos y convicciones y favoreciendo la interiorización de estas buenas prácticas, más allá de los controles. En definitiva, se trata de desarrollar un ethos profesional, un carácter que se adentre en la lógica propia de la actividad. Recuérdese que en el caso de los servicios públicos, éstas son actividades cooperativas, recurrentes e institucionalizadas y donde, en consecuencia, la cultura institucional ejerce una enorme influencia en las percepciones y elecciones de los servidores públicos. Así, el entorno de expectativas y roles afecta y explica una parte importante de las conductas finales de los implicados (stakeholders).

\subsection{Protección y prevención}

Los códigos de buenas prácticas contribuyen a defender la actividad y los bienes internos que la caracterizan frente a presiones externas (económicas, mediáticas...). Constituyen por ello mecanismos protectores de la actividad que, en el caso de las organizaciones del sector público, operan como garantía de imparcialidad. Asimismo, son un valioso instrumento de prevención de malas prácticas, abusos y corrupción pues cuando intervienen los tribunales de justicia, el daño a la organización y al servicio público ya está hecho y es muy difícil revertirlo (Jiménez, 2017a). Los códigos son 
instrumentos de autorregulación con una dimensión eminentemente preventiva, frente al carácter represivo o disuasorio de los marcos jurídicos. Ya hemos hablado del carácter básicamente orientativo de los códigos de buenas prácticas dentro del conjunto de instrumentos y herramientas para fomentar la excelencia y unos altos estándares de conducta en el seno de las organizaciones del sector público (infraestructura ética).

\subsection{Ejemplaridad}

Los códigos de buenas prácticas acrecientan el prestigio de una actividad, dignificándola socialmente y estableciendo condiciones de ejemplaridad (la fuerza de lo que es como debería ser). Por ello, estos códigos son modelos, patrones, que marcan pautas deseables en la configuración y desarrollo de una actividad. De aquí que necesariamente los códigos hayan de ser públicos, proporcionando información no solo a los profesionales sino también a la sociedad. Sin duda, la ejemplaridad constituye uno de los valores esenciales para la dignificación de las actividades del servicio público y la restauración de la confianza entre ciudadanos y servidores públicos. Al gobernante y al gestor público solo le cabe practicar con el ejemplo (Gomá, 2009).

\subsection{Confianza}

Los códigos de buenas prácticas, en tanto que elementos fundamentales de la infraestructura ética de las organizaciones y, muy en particular, de las del sector público, contribuyen al control social de las actividades profesionales, políticas y de la administración, estableciendo prácticas y pautas de conductas deseables y, a contrario, señalando y censurando la mala praxis en dichos ámbitos. Pero además, como hemos dicho, contribuyen a generar hábitos, convicciones y cambio de cultura en las organizaciones, mediante la replicación y repetición de experiencias positivas, lo que genera confianza, capital social e incentivos para cooperar. No es posible mantener una sociedad compleja sin la confianza y, por tanto, una función fundamental de los códigos de buenas prácticas es la de generar y restaurar confianza entre los ciudadanos y las organizaciones, especialmente las instituciones políticas, en un momento de máximo descrédito de las mismas (Ausín, 2015).

\section{Valoración}

Acabamos de presentar una serie de importantes funciones que los códigos de buenas prácticas pueden ejercer con respecto a diferentes actividades y contextos y, en concreto también, en lo que se refiere a las organizaciones del sector público: el reconocimiento de la misión de la actividad, la generación de conocimiento, aprendizaje e innovación sobre la misma, la protección frente a presiones externas, la prevención de malas prácticas, la confianza y la ejemplaridad. Todos estos elementos tienen un innegable carácter positivo con relación a la actividad y abundan en una consideración favorable hacia los códigos de buenas prácticas.

Sin embargo, en muchas ocasiones se han percibido estos instrumentos como la defensa de unos privilegios de grupo, que refuerzan el poder de determinadas actividades o agrupaciones profesionales. Y es que la innegable función de protección de la actividad a la que nos hemos referido no debería eludir el sometimiento de la misma a las normas jurídicas legítimamente establecidas. Así, en el debate en la II República española sobre la abolición de los Tribunales de Honor se adujo que, por principio, en un Estado de Derecho no era aceptable el mantenimiento de una justicia privada de carácter gremial -a lo que se añadía el irregular procedimiento que normalmente seguían estas instituciones y la falta de equidad derivada tanto de la imprevisión de las causas como de la no posibilidad de recurrir contra sus decisiones. Nicolás Pérez Serrano decía, por su parte, con respecto a los Tribunales de Honor: 
“... nacidos para velar por el honor profesional, degeneraron muchas veces, sirviendo su secreto y las escasas garantías de sus procedimientos para satisfacer pasiones políticas, rencores de Cuerpo o estímulos de codicia. Justo es reconocer también que en ocasiones sirvieron para librar a verdaderos delincuentes de las penas que su conducta merecía. En general, pues, eran instrumentos de inmenso peligro y de harto discutible provecho: muy pocos sentirán su desaparición" (tomado de DomínguezBerrueta, 1992). Más recientemente, Alejandro Nieto ha criticado la falta de precisión de los códigos de ética y de conducta, que supondrían una quiebra del principio de legalidad en tanto en cuanto pueden dar lugar a expedientes disciplinarios o evaluaciones desfavorables (Nieto, 2008).

En esta línea crítica, también se ha aducido que el concepto de "buenas prácticas" remite a una estrategia de despolitización y a un enfoque pragmático, cientificista, basado en la experiencia y que reniega de la política (Hammersley, 2013). Un enfoque que supone un modo de capturar la realidad de una actividad obviando consideraciones políticas y de poder ${ }^{4}$, subrayando la pertinencia de prescindir de los prejuicios, separando lo técnico de lo político y defendiendo la aplicación de las "buenas ideas", vengan de donde vengan ideológicamente, en lo que Bourdieu (2001) llama "políticas de despolitización". Para esta crítica del concepto de "buenas prácticas" hay preguntas previas ineludibles que han de responderse: ¿Qué es una "buena práctica"? ¿Para quién? ¿Quién evalúa y decide su bondad? ¿Cómo? (Shore y Wright, 2015).

Asimismo, una sombra de desconfianza y escepticismo cae sobre los códigos de buenas prácticas, dado que en muchas ocasiones han tenido un carácter meramente retórico y vacío, cuando no descaradamente publicitario, pero sin ningún impacto sobre la calidad y excelencia de la actividad. Peor aún, este tipo de códigos meramente programáticos, que conciben la ética como mera cosmética, han generado mayor frustración y desconfianza y han ejercido como coartada, en muchos casos, de malas prácticas (un sector con un amplio elenco de códigos de buenas prácticas es el de la banca, por ejemplo).

\section{Requisitos}

Si los códigos de buenas prácticas aspiran a realizar las relevantes funciones que hemos formulado (reconocimiento, pedagogía, protección, prevención, confianza y ejemplaridad), evitando las críticas a las que también nos hemos referido (gremialismo, cientificismo, despolitización y desconfianza), habrán de satisfacer una serie de requisitos mínimos que explicitamos a continuación:

\subsection{Participación}

Los códigos se han de desarrollar e implementar desde las propias organizaciones, desde quienes llevan a cabo la actividad, con todo el asesoramiento externo que se precise y, si fuera posible, con la intervención de los legos, de la ciudadanía. En el caso de los códigos de buenas prácticas para el sector público, todos los actores de las políticas públicas (políticos, técnicos, asociaciones, individuos) definirían sus necesidades, reconociendo las necesidades de los demás, cooperando en el establecimiento de los satisfactores, compartiendo soluciones e implicándose, en última instancia, en la acción, -interviniendo en el balance social de los resultados (coresponsabilidad, co-gestión). Desde esta estrategia de abajo-arriba (bottom-up) se

4 Según Alejandro Nieto, este tipo de instrumentos puede permitir a determinados agentes sociales con poder hacerse resistentes a la influencia política e incluso dominarla (Nieto 2008: 272). 
evitaría la crítica de la "despolitización" y de la reducción de las "buenas prácticas" a consideraciones meramente técnicas y cuasi-científicas (tecnocráticas), favoreciendo la aceptación e interiorización de las mismas, por mor de su mayor legitimidad (Schwenke, 2007). Preguntas como las mencionadas (¿Qué es una "buena práctica"? ¿Para quién? ¿Quién evalúa y decide su bondad? ¿Cómo?) deberían ser respondidas desde procesos con la máxima participación y transparencia.

En todo caso y como mínimo, no habría que "importar" documentos ajenos, elaborados por otras organizaciones, para otros contextos, o por comisiones ajenas al propio entramado de la organización, como ha ocurrido en algunos de los llamados "procesos de modernización" de las administraciones públicas.

\subsection{Flexibilidad}

Los códigos han de ser documentos abiertos y flexibles (Verbos et al., 2007). No son las tablas de la ley inmutables y fijas. La evolución de las demandas de la sociedad, las nuevas exigencias, circunstancias y comportamientos, hacen que los códigos se vayan reformando y modificando, convirtiéndose en un instrumento "vivo" para la mejora continua de los estándares éticos del servicio público y el fortalecimiento de la confianza pública. Esta concepción flexible de los códigos de ética pública implica procesos de evaluación de su despliegue y aplicación, tanto evaluación interna, a través de su organismo de monitorización (véase más adelante), como externa, a cargo de instituciones evaluadoras.

\subsection{Adhesión}

Debe quedar claro a quién afecta el código y cómo se establece la fuerza vinculante del mismo, pues no se deriva del cumplimiento de las leyes (como se ha dicho, va de suyo la obligación de cumplir las leyes). Por ello son necesarios mecanismos de adhesión al código que sean sencillos, transparentes y públicos de modo que, además, queden claros los compromisos y responsabilidades que se asumen al adoptar un código de buenas prácticas.

\subsection{Canales}

Se precisan procedimientos, medios y protocolos accesibles tanto para las denuncias como para las consultas relativas a los códigos de buenas prácticas. Para ello, se garantizará la confidencialidad, cuando sea necesario, y se atenderá especialmente a la protección de los denunciantes (whistleblowers).

\subsection{Monitorización}

Es imprescindible la existencia de un sistema de seguimiento de los códigos que, además, ofrezca información pública y accesible sobre la actividad desarrollada en torno a estos documentos. Esto es fundamental para evitar la acusación de gremialismo. Para ello, se establecen comisiones o comités de ética que impulsan, coordinan y realizan el seguimiento de los códigos de buenas prácticas en las organizaciones y que actúan como órganos de garantía del cumplimiento y desarrollo de los códigos. En este punto, no es baladí la cuestión sobre cómo se configuran estos instrumentos de seguimiento: ¿Cuál es la composición de las comisiones? ¿Cómo se escoge a sus miembros? ¿Cuáles son sus atribuciones?

Entre sus cometidos concretos están el atender quejas y denuncias, resolver consultas y dilemas que se presentan a los concernidos en la actividad, formular propuestas y recomendaciones (ya hemos dicho que los códigos son instrumentos vivos 
y flexibles) y promover la difusión y la formación a través de cursos, seminarios, conferencias, etc. En algunos casos, se incluyen entre sus cometidos el de sancionar en caso de incumplimiento del código. Este es un asunto muy debatido con relación a los códigos de buenas prácticas, pues implica traspasar su carácter orientador y entrar en su valor disciplinario. La pregunta clave es si los códigos de buenas prácticas necesitan sanciones por incumplimientos para cambiar conductas y malas prácticas ${ }^{5}$.

\subsection{Difusión}

Para que los códigos de buenas prácticas contribuyan efectivamente al cambio de cultura institucional en una determinada organización o servicio público ha de promoverse no solo su conocimiento y difusión entre los directamente implicados por el mismo (y la sociedad en su conjunto), sino que se debe profundizar en la formación y comprensión de sus valores, principios, procedimientos y desarrollo a fin de favorecer la interiorización de los mismos para la mejora continua del clima ético en las instituciones y organizaciones. Cursos, seminarios, conferencias y el uso de tecnologías de la información y la comunicación -como páginas web, apps, redes sociales y otros recursos electrónicos- son fundamentales para esta extensión de la cultura ética y de las buenas prácticas.

\section{Conclusiones}

Como hemos dicho, las "buenas prácticas" se refieren a aquellas experiencias, procesos y actividades que resultan aconsejables en un determinado ámbito, público o privado, porque han arrojado resultados positivos y han demostrado su éxito y utilidad en dicho contexto, de modo que merece la pena que se repitan y sean compartidas por el mayor número de implicados que puedan adoptarlas, para lo cual se sistematizan a través de su formulación en códigos u otros documentos. Tienen la pretensión de incidir en el ethos (carácter) de la actividad, generando hábitos, convicciones y cambio de cultura organizacional. Los códigos de buenas prácticas se mueven entre la tradición y la innovación y muchas veces se han podido percibir como instrumentos de defensa de unos privilegios de grupo o gremio, favoreciendo una estrategia de despolitización de las actividades implicadas y adoptando un carácter meramente retórico y vacío. Sin embargo, los códigos de buenas prácticas ejercen una serie de importantes funciones: el reconocimiento de la misión de la actividad, la generación de conocimiento, aprendizaje e innovación sobre la misma, la protección frente a presiones externas, la prevención de malas prácticas, la confianza y la ejemplaridad. Para satisfacer estas importantes tareas, los códigos de buenas prácticas deberán cumplir una serie de requisitos mínimos: participación, flexibilidad, canales de comunicación, sistemas de adhesión y monitorización y una estrategia de difusión y formación. Por ello, los códigos de buenas prácticas suponen un notable compromiso para su correcta y eficaz implantación en las organizaciones.

\footnotetext{
5 Esta cuestión remite a los dos tipos de estrategias que se distinguen a la hora de abordar procesos de mejora y excelencia en las organizaciones del sector público: la "ruta fácil" (low roads) y la "ruta difícil" (high roads). La primera atiende a los controles externos y sancionadores de las conductas, estableciendo normas y procedimientos detallados para reducir las malas prácticas. La "difícil" se centra en la autorregulación, los controles internos, la formación y el cambio de cultura organizacional (OCDE 1997). Una distinción similar es la que establece Rafael Jiménez Asensio al hablar de dos caminos para luchar contra la corrupción: el "rápido", mediante leyes represivas, y el "lento", a través de la autorregulación y de marcos de integridad institucional (Jiménez, 2017a). Para este autor, "... las consecuencias traumáticas de la gestión de los códigos deben ser una excepción. Lo habitual es que las políticas de integridad se construyan en clave positiva y siempre con carácter preventivo." (Jiménez, 2017a: 93).
} 
Bibliografía

ALGUACIL, J. (2012), “Administración relacional”, Eunomía, 2, pp. 152-160.

AUSíN, T. (2012), "Ética de las administraciones", Eunomía, 1, pp. 141-147.

AUSíN, T. (2015), "Ética pública para generar confianza", Revista Vasca de Gestión de Personas y Organizaciones Públicas 9(2015), pp. 30-39.

BOURDIEU, P. (2001), "Contra la política de despolitización", Ecología política, 21, pp. 99-102.

COMISIÓN EUROPEA (2004), Manual sobre la integración para responsables de la formulación de políticas y profesionales, Comunidades Europeas, Bruselas.

DIEGO BAUTISTA, O. (2013), Ética para gobernar, Senado de la República, México.

DOMÍNGUEZ-BERRUETA, M. (1992), "De nuevo sobre los Tribunales de Honor", Revista Vasca de Administración Pública, 33, pp. 27-90.

ECHEVERRÍA, J. (2017), El arte de innovar, Plaza y Valdés, Madrid.

GOMÁ, J. (2009), Ejemplaridad pública, Taurus, Madrid.

HAMMERSLEY, M. (2013), The myth of research-based policy \& practice, Sage, London.

JIMÉNEZ ASENSIO, R. (2017a), Cómo prevenir la corrupción. Integridad y transparencia, La Catarata, Madrid.

JIMÉNEZ ASENSIO, R. (2017b), "Una mirada 'comparada' sobre algunas experiencias de códigos éticos y de conducta en la función pública", Revista Vasca de Gestión de Personas y Organizaciones Públicas 13, pp. 58-79.

LAFUENTE, A., \& HORRILLO, P. (2017), "Cómo hacer un prototipo", EducaLab, disponible en la siguiente dirección electrónica: http://laaventuradeaprender.educalab.es/guias/como-hacer-un-prototipo (fecha de consulta: 13 de abril de 2018).

LÍBANO BERISTAIN, A. (2011), "Códigos deontológicos". En: ROMEO CASABONA, C. (ed.), Enciclopedia de Bioderecho y Bioética, Tomo I, Granada, Comares, pp. 375-380.

LONGO, F. y ALBAREDA, A. (2015), Administración pública con valores. Instrumentos para una gobernanza ética, INAP, Madrid.

NIETO, A. (2008), El desgobierno de lo público, Ariel, Barcelona.

OCDE (1997), La ética en el servicio público, MAP, Madrid.

OCDE (2000), Confianza en el gobierno. Medidas para fortalecer el marco ético en los países de la OCDE, París.

SCHWENKE, C. (2007), Formulating and Implementing Effective Code of Ethics: Guidance Manual for Public Institutions, Inter-American Development Bank, Washington DC.

SHORE, C. y WRIGHT, S. (2015), "Governing by numbers: audit culture, rankings and the new world order", Social Anthropology, 23(1), pp. 22-28.

VERBOS, A.K. et al. (2007), "The Positive Ethical Organization: Enacting a Living Code of Ethics and Ethical Organizational Identity", Journal of Bussiness Ethics, 76(1), pp. 17-33.

VILLORIA, M. \& IZQUIERDO, A. (2015), Ética pública y buen gobierno, Tecnos, Madrid.

VON HIPPEL, E. (2005), Democratizing Innovation, MIT Press, Cambridge MA. 